\title{
Mechanism of Gas-Phase Aldose-Ketose Isomerization: A Study Using Tandem Mass Spectrometry and Theoretical Calculations
}

\author{
Ya-Jun Zheng, Andreas Staempfli, ${ }^{*}$ and Julie A. Leary \\ Department of Chemistry, University of California, Berkeley, California, USA
}

Fast-atom bombardment tandem mass spectrometry and the semiempirical molecular orbital method were used to investigate the mechanism of gas-phase aldose-ketose isomerization process in lithiated 1,3 linked disaccharide isomers. Both the 1,3 hydrogen shift and hydride transfer mechanisms were investigated. Our experimental and theoretical calculations support the latter. The hydride transfer mechanism in these lithium-coordinated systems is similar to the xylose isomerase catalyzed aldose-ketose isomerization. (J Am Soc Mass Spectrom 1993, 4, 943-948)

$\mathrm{F}$ ast-atom bombardment (FAB) tandem mass spectrometry (MS/MS) of lithium-coordinated oligosacchatides has been shown to be an effective method of determining the linkage position of various isomeric oligosaccharides [1-3]. Collisioninduced dissociation (CID) of either the monolithiated $\left([\mathrm{M}+\mathrm{Li}]^{+}\right)$or clilithiated $\left([\mathrm{M}+2 \mathrm{Li}-\mathrm{H}]^{+}\right)$species produces product ions of the isomers which are different and very specific to the linkage position. In these earlier studies, we postulated that to observe the different losses for each isomeric disaccharide, the reducing ring hemiacetal must first undergo ring opening to produce the hydroxy aldehyde [2]. Subsequent retroaldol rearrangement then gives rise to specific two, three, and/or four carbon neutral losses from the reducing ring. In one particular case, the alpha and beta 1,3 linked disaccharides, transposition of the carbonyl function from $\mathrm{C}-1$ to $\mathrm{C}-2$ of the hydroxy aldehyde is required prior to the retro-aldol rearrangement $[2,3]$. Labeling studies employing ${ }^{18} \mathrm{O}$ and ${ }^{2} \mathrm{H}$ indicated that the 1,3 linked disaccharide must rearrange to transpose the carbonyl to the $\mathrm{C}-2$ position prior to the retro-aldol reaction [3] to observe the characteristic three carbon chain neutral loss $\left(\mathrm{C}_{3} \mathrm{H}_{4} \mathrm{D}_{2} \mathrm{O}_{3}\right)$, as shown in Scheme I (Figures 2 and 3 , discussed later, provide the accurate metal-binding site).

The data presented here are aimed at investigating the mechanism(s) required for this carbonyl transposition. Tandem mass spectrometry in combination with semiempirical calculations were used to determine the

\footnotetext{
${ }^{*}$ Currently at Nestec Ltd., SA Research Centre, Lausanne, Switzerland.

Address reprint requests to Julie A. Leary, Department of Chemistry, University of California, Berkeley, CA 94720.
}

cnergetics involved in the pathway(s) which lead to migration of the carbonyl. There are two important criteria which are accepted in discussing both the theoretical and experimental data for this study. These are: (1) reducing ring opening followed by the retro-aldol rearrangement takes place for lithium-coordinated saccharides which undergo MS/MS experiments; and (2) carbonyl migration from $\mathrm{C}-1$ to $\mathrm{C}-2$ of the reducing ring must occur for the lithiated 1,3 linked disaccharides before dissociation can occur [3]. The first assumption is based on extensive labeling experiments $[2,3]$.

Two reaction pathways were investigated. Both involve ring opening, but differ in the aldehyde-ketone isomerization step. In reaction pathway $A$, the isomerization follows a keto-enol tautomerization via a $1,3-\mathrm{H}$ shift while reaction pathway $B$ involves a proton transfer from the C-2 hydroxyl to the carbonyl oxygen ( $C-1)$ followed by a hydride transfer from $\mathrm{C}-2$ to $\mathrm{C}-1$ (Scheme II). Keto-enol tautomerization is well known in solution chemistry and this reaction can be either base- or acid-catalyzed [4-6]. However, there is little evidence that this reaction occurs in the gas phase. The pure enol and keto forms are usually very stable if catalysts such as acids or base are absent [7]. The simplest enol, vinyl alcohol, has been prepared in the gas phase and has been shown to have a lifetime of 30 minutes [8]. Theoretical calculations predicted a very high activation barrier for the vinyl alcohol-acetaldehyde transformation [9]. Because of this large barrier, those authors suggested that the observed vinyl alcoholacetaldehyde transformation must be due to some other mechanism. Although there have been no experimental gas-phase data measuring the activation energy of the keto-enol isomerization, a value for the $1,3-\mathrm{H}$ shift 


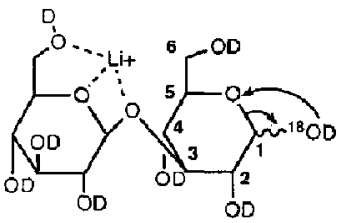

$\beta$ 1,3 linked disaccharide

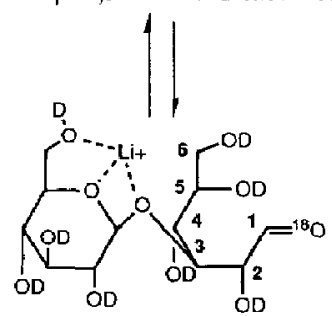

Aldose-Ketose Isamerization
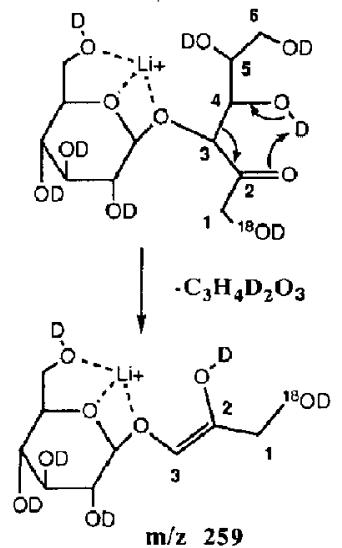

Scheme I. Proposed mechanism of carbonyl migration and retro-aldol rearrangement.

in propene $(87.7 \mathrm{kcal} / \mathrm{mol})$ has been reported [10]. This reaction also involves a very high activation barrier in the gas phase.

Pathway B, the hydride transfer mechanism, is related to that proposed for xylose isomerase-catalyzed aldose-ketose isomerization reactions [11, 12]. Xylose isomerase is a metalloenzyme which requires divalent metal ions (usually $\mathrm{Mn}^{2+}$ or $\mathrm{Mg}^{2+}$ ) for catalytic activity. In the X-ray crystal structure there are two metal ions in the active site; one which binds to $\mathrm{O} 2$ and $\mathrm{O} 4$ of xylose [11]. The other metal coordinates a water molecule (as the hydroxide form during catalytic process) which acts as a base to deprotonate the hydroxyl hydrogen at $\mathrm{C} 2$. The mechanism of xylose isomerase catalyzed aldose-ketose isomerization is shown in Scheme III. For acid-catalyzed aldose-ketose isomerizations, there is also evidence that the hydride transfer mechanism plays a very important role $[12,13]$.

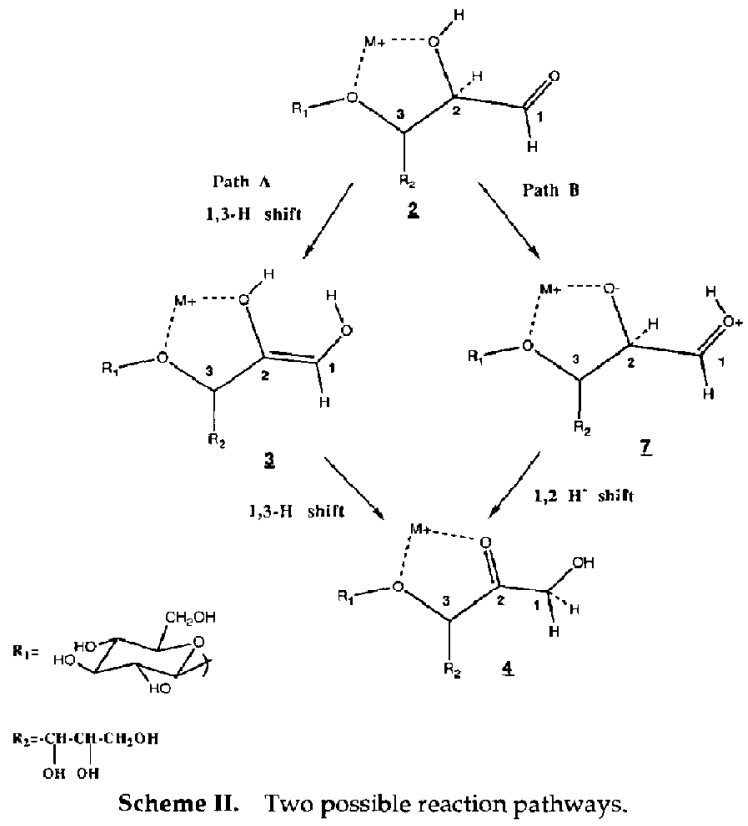

Experimental

\section{Mass Spectrometry}

Mass spectra were obtained using a VG ZAB2-EQ mass spectrometer (VG Analytical, Greater Manchester, UK) of $\mathrm{BEOQ}$ geometry [o is a radiofrequency<smiles>O=CC1C(NCO)CCC(CO)C1O</smiles>

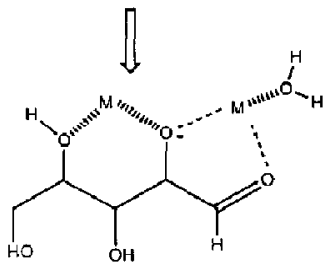<smiles>C=CCOC(=O)C1=COC(O)C(CO)C1O</smiles>

Scheme III. Mechanism for xylose isomerase catalyzed aldose-ketose isomerization. For simplicity, the active site residues and metal-bound ligands are omitted. 
(RF)-only octapole collision cell fabricated in our laboratory and replaces the conventional quadrupole cell in this particular instrument]. The instrument is equipped with three reaction regions: the first is a reaction region for $B / E$ linked scanning; the second reaction region is used for mass-analyzed ion kinetic energy spectrometry (MIKES) scanning; and the third region is an RFonly octapole collision cell followed by a quadrupole mass analyzer. The precursor ions, $\left[\mathbf{M}+\mathrm{Li}^{+}\right.$, were generated using $20-\mathrm{keV}$ cesium ion bombardment with approximately $1.0 \mu \mathrm{A}$ current. Both unimolecular (no collision gas) and collision-induced dissociation (CID) spectra were obtained utilizing all three reaction regions. For high energy ( $8 \mathrm{keV}$ ) $\mathrm{B} / \mathrm{E}$ linked scanning and MIKES CID data, the collision gas used was helium and the corresponding gas pressure was $1 \times 10^{-6}$ torr in each case. Product ion spectra generated using the octapole collision cell were collected using argon as collision gas at a gas pressure of $1-2 \times 10^{-6}$ torr. Collision energies ranged from 10 to $30 \mathrm{eV}$ (laboratory frame of reference).

The 1,3 linked isomers, laminaribiose and nigerose, were purchased from Sigma Chemical Co. (St. Louis, $\mathrm{MO}$ ) and prepared as described previously [2]. Briefly, $1 \mu \mathrm{L}$ of the disaccharide (1.5 $\mathrm{nM}$ aqueous) was diluted in $3 \mu \mathrm{L}$ of a 50:50 mixture of dithioerythreitol: dithiothreitol. Three microliters of $1.5 \mathrm{nM}$ aqueous lithium hydroxide or lithium carbonate was then added to the above mixture. From this, $1 \mu \mathrm{L}$ of solution was deposited on the probe tip.

\section{Theoretical Calculations}

The semiempirical molecular orbital calculations were carried out using the MNDO [14] method as implemented in the MOPAC 6.0 program. * Previous calculations have shown that MNDO gives reliable results for lithium-coordinated saccharides $[2,3]$. The initial disaccharide geometry was taken from the Cambridge Data Base and the lithium-coordinated structure was found by using the same procedure as described previously $[2,3]$. Geometries were fully optimized and the gradient was normally reduced to below 1.0 (in most cases, less than 0.1 ). Transition states were located by the reaction coordinate method and further refined using the gradient optimization method (NLLSQ in MOPAC 6.0).

It is well known that semiempirical methods such as MNDO and AM1 are parameterized to reproduce experimental data such as heat of formation, geometrical parameters (bond length, bond angle, and dihedral angle), ionization potential, dipole moment, etc. In spite of the fact that these semiempirical methods were parameterized for neutral compounds, they perform reasonably well for charged species because electron

*Available from Quantum Chemistry Program Exchange, program no. 455. Department of Chemistry, Indiana University, Blooming ton, IN 47405. correlations have been built in via parameterization procedures. A detailed discussion on these methods is provided elsewhere $[15,16]$ in addition to a comprehensive study on proton affinity reported by Dewar and Dieter [17].

As an additional comparison of calculated versus experimental values, we investigated MNDO calculations of the following proton transfer reaction for which experimental data already exist. (The experimental data are calculated from the experimental heats of formation provided in refs 14 and 18.) We observed that the calculated value agrees very well with the experimental value.

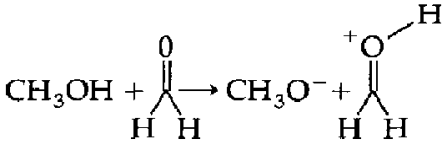

$$
\begin{aligned}
& \text { MNDO Calculated: } \quad \Delta \mathrm{H}=206 \mathrm{kcal} / \mathrm{mol} \\
& \text { Experimental: } \quad \Delta \mathrm{H}=205 \mathrm{kcal} / \mathrm{mol}
\end{aligned}
$$

\section{Results and Discussions}

\section{Unimolecular and CID Experiments}

In a previous study we showed, using MS/MS, that ${ }^{18} \mathrm{O}$ and ${ }^{2} \mathrm{H}$ labeled laminaribiose produced spectra which indicated loss of a three-carbon neutral fragment after transposition of the carbonyl from C-1 to $\mathrm{C}-2$ of the reducing ring [3]. The resulting ion is characteristic for determining the linkage position of oligosaccharide isomers since it is observed as the only ring cleavage ion for monolithiated 1,3 linked oligosaccharides. This three-carbon neutral loss is not observed for the 1,2-or 1,4-linked isomers. To learn more about the carbonyl transposition, we began looking at the high and low energy CID spectra for two isomeric 1,3 linked disaccharides, nigerose (Glc- $\alpha-(1,3)$-Glc), and laminaribiose (Glc- $B-(1,3)-G l c)$. Table 1 lists the ions observed in the metastable and CID spectra of nigerose as collected from the first (B/E), second (MIKES), and third (octapole) collision regions. Similarly, Table 2 lists the respective ions collected for laminaribiose. The only difference in these two compounds is the stereochemistry of the glycosidic oxygen, that is, alpha versus beta. Scheme IV provides diagrammatic information as to the origin of the ions listed in Tables 1 and 2.

It is interesting to note that in these spectra, all ions present result from stable neutral losses (and therefore via various rearrangements) rather than by direct bond cleavage. (This has been observed in our previous investigations and has been discussed in detail [1-3].) These data suggest that the precursor ions are of relatively low internal energy. For instance, it has been shown for protonated ethanol, that at high energies, one sees loss of hydrogen and methyl radicals during CID of nonthermal closed shell species [19]. This has also been shown to be true for high energy CID of protonated oligosaccharides [20]. However, this is not 
Table 1. Product ions observed in the tandem mass spectrum of $[\mathrm{M}+\mathrm{Li}]^{\dagger}$ of nigerose*

\begin{tabular}{lccc}
\hline$m / z$ & $B / E^{a}$ & MIKES $^{b}$ & OCTA $^{c}$ \\
\hline Meta $^{d}$ & & & \\
331 & $100^{\dagger}$ & 100 & 100 \\
259 & - & 2.7 & 10 \\
229 & - & - & - \\
187 & - & - & 65 \\
169 & - & 100 & 8 \\
$C_{10}$ & & 4.6 & 100 \\
331 & 100 & 1.5 & 4.2 \\
259 & - & 6.3 & 70 \\
229 & 1 & 2.7 & 29 \\
187 & 6 & & \\
169 & 2.3 & & \\
\hline
\end{tabular}

* Data are collected from three reaction regions.

- First field-free region using $B / E$ linked scan.

${ }^{b}$ Second field-free region using the MIKES scan.

'MS/MS data collected using octapole collision cell for CID or metastable scans

dData collected under metastable (unimolecular) conditions.

Data collected using collision gas (CID).

${ }^{t}$ Data are presented as percent abundance relative to $m / z 331$.

Table 2. Product ions observed in the tandem mass spectrum of $[\mathrm{M}+\mathrm{Li}]^{+}$of laminaribiose*

\begin{tabular}{|c|c|c|c|}
\hline$m / z$ & $\mathrm{~B} / \mathrm{E}^{\mathrm{a}}$ & MIKES $^{b}$ & OCTA $^{\circ}$ \\
\hline \multicolumn{4}{|l|}{ Meta $^{d}$} \\
\hline 331 & $100^{\prime}$ & 100 & 100 \\
\hline 259 & - & 4.7 & 14.4 \\
\hline 229 & - & - & - \\
\hline 187 & - & 2.3 & 27 \\
\hline 169 & -- & - & 12 \\
\hline \multicolumn{4}{|l|}{$\mathrm{CIO}^{\circ}$} \\
\hline 331 & 100 & 100 & 100 \\
\hline 259 & - & 8.1 & 5.8 \\
\hline 229 & 1.8 & 3.5 & - \\
\hline 187 & 7.2 & 8.5 & 86 \\
\hline 169 & 6.0 & 4.6 & 141 \\
\hline
\end{tabular}

*Data are collected from three reaction regions

- First field-free region using $\mathrm{B} / \mathrm{E}$ linked scan.

becond field-free region using the MiKES scan.

cMS/MS data collected using octapole collision cell for CID or metastable scans.

Data callected under metastable (unimolecular) conditions.

e Data callected using collision gas $(\mathrm{CID})$.

f Data are presented as percent abundance relative to $m / z 331$

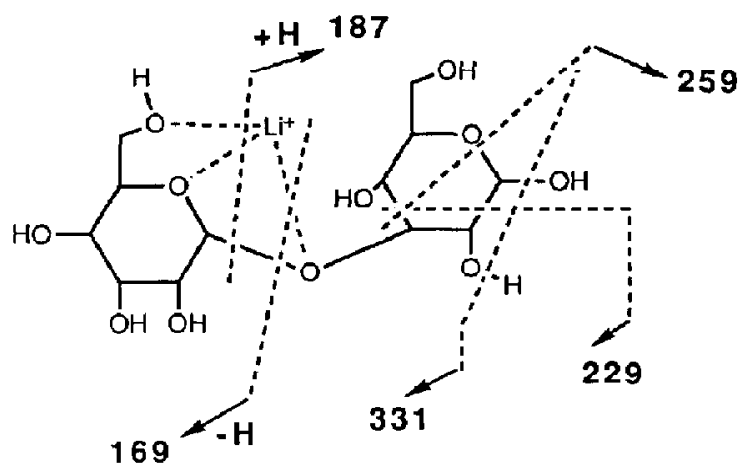

Scheme IV. Origination of ions detected. what we see in either the unimolecular or CID spectra generated from all three reaction regions of the instrument. Neither the $B / E$ linked scan data nor the MIKES data indicate the presence of ions resulting from single homolytic bond cleavage. Since the MS/MS octapole scans represent low energy collisions, it is not surprising that one sees only rearrangement ions in this case.

A more interesting observation is the noticeable dissimilarity among the spectra of each compound with regard to the ion $m / z 259$ (see Tables 1 and 2). The unimolecular dissociation spectrum from the $B / E$ linked scan experiment primarily shows ions resulting from the loss of water with no appreciable amounts of any other ions. The CID spectrum from this same collision region shows both glycosidic cleavage ions $(m / z 169$ and 187$)$ as well as another type of ion with $m / z 229$. However, the ion at $m / z 259$ is still noticeably absent and yet both the MIKES and OCTA spectra indicate the presence of this ion under unimolecular and CID conditions. This difference is not observed with other lithium disaccharides which do not require carbonyl migration prior to the retro-aldol rearrangement. For example, the $[\mathrm{M}+\mathrm{Li}]^{+}$ions from the lithiated 1,4 linked isomers, lactose and maltose, produce product ions with $m / z 289$ (two carbon chain neutral loss via the retro-aldol reaction) in all three reaction regions. We suggest that these differences are because the time frame for carbonyl migration followed by the retro-aldol reaction exceeds the time required for detection of ions in the $B / E$ linked scan spectra. If this were the case, then based upon the equation $t=d \sqrt{\mathrm{m} / 2 \mathrm{ZeV}}$ [21], the process of carbonyl migration followed by retro-aldol rearrangement fails somewhere between $1.7 \mu \mathrm{sec}$ [time ion is expected to reach field-free region (FFR) 1 in the $B$ /E experiment] and $55 \mu$ sec (time ion reaches FFR-2 and is actually observed in the MIKES experiment in our ZAB2-EQ instrument). Unfortunately, there are no totally reliable experiments which we can perform using our current instrument to further prove this hypothesis. We did try lowering the accelerating voltage from $8 \mathrm{kV}$ to $4 \mathrm{kV}$ for the $\mathrm{B} / \mathrm{E}$ linked scan experiment and looked for differences in the spectra, but observed none. This is not surprising, given the fact that when $V$ is changed from 8 to 4 in the above equation, $t$ only changes by a very small amount, that is, $1.7 \mu \mathrm{sec}$ at 8 $\mathrm{kV}$ versus $2.4 \mu \mathrm{sec}$ at $4 \mathrm{kV}$. This is a very small difference, considering $t$ is $55 \mu$ sec in the MTKES experiment.

Although the time frame of the experiment cannot be unambiguously determined, we can address the mechanism of carbonyl migration in the gas phase by utilizing semiempirical calculations as described below.

\section{Energetics of Pathways $A$ and $B$}

Two reaction pathways were investigated by semiempirical calculations to determine the more likely mech- 
anism for carbonyl migrations. MNDO-calculated reaction profiles for both the 1,3-H shift (Pathway A) and the hydride transfer (Pathway B) mechanisms are shown in Figure 1. The calculated structures for the hemiacetal 1 and the aldehyde form 2 are shown in Figures 2 and 3 , respectively. (All other structures, along with heats of formation, are provided as supplementary material.)

The reaction associated with Pathway $B$ involves an initial proton transfer from the $\mathrm{C}-2$ hydroxyl group to the $\mathrm{C}-1$ carbonyl oxygen with a calculated barrier of $56.5 \mathrm{kcal} / \mathrm{mol}$. From this first transition state (TS), 6 the resulting zwitterionic intermediate 7 undergoes a rapid hydride transfer with a barrier of $12.4 \mathrm{kcal} / \mathrm{mol}$ to reach the second transition state 8 . Subsequent rearrangement to the final keto structure is then exothermic by $58.1 \mathrm{kcal} / \mathrm{mol}$.

In contrast to the hydride transfer, calculations for the keto-enol tautomerization via a 1,3-H shift indicate a much larger barrier $(77.4 \mathrm{kcal} / \mathrm{mol})$ associated with formation of the TS for enolization 5 . The enol intermediate 3, although more stable than the zwitterionic intermediate for the hydride transfer mechanism, must then overcome another large barrier $(89.0 \mathrm{kcal} / \mathrm{mol})$ before rearranging to the final keto structure. Thus, the overall barrier for the $1,3-\mathrm{II}$ shift is $95.2 \mathrm{kcal}$ versus $66.0 \mathrm{kcal}$ for the hydride transfer (beginning from the hemiacetal at $-398.2 \mathrm{kcal}$, to the final keto structure). Patlıway B is clearly favored over Pathway A.

One might argue that since we do not know the internal energy of these ions, either pathway may be possible. This, in fact, may be true. However, because the 1,3-H shift requires significantly more energy than the hydride transfer mechanism, the latter is clearly

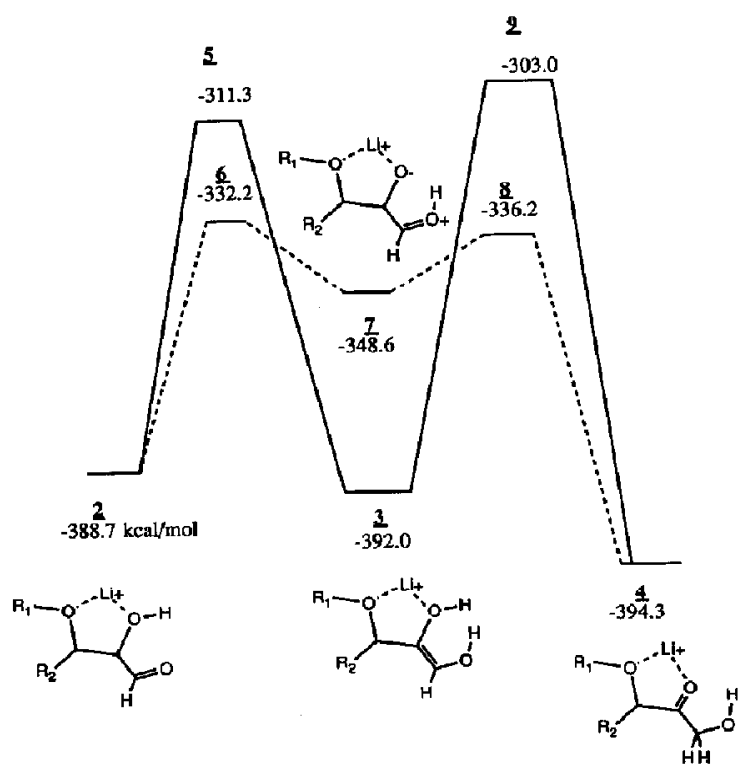

Figure 1. The calculated reaction profiles for Path $\mathrm{A}$ (solid line) and Path B (broken line) (see Scheme II).

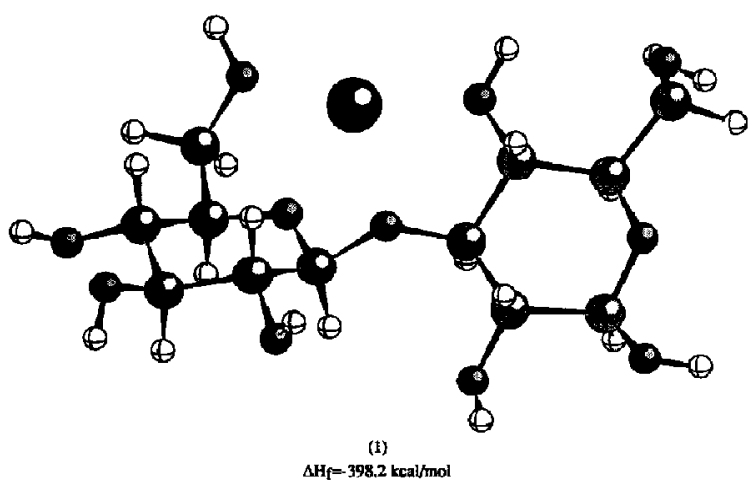

Figure 2. The calculated structure for the lithium coordinated hemiacetal (1).

favored and is expected to compete significantly with the 1,3-H shift. In fact, if $95 \mathrm{kcal}$ were available for the $1,3-\mathrm{H}$ shift, then some portion of that energy should also be available for simple bond cleavage. We should have seen evidence for these reactions in the corresponding tandem mass spectra, given the fact that simple $\mathrm{C}-\mathrm{O}$ bond cleavage is approximately 90 $\mathrm{kcal} / \mathrm{mol}$ for alcohols and $80 \mathrm{kcal} / \mathrm{mol}$ for ethers [22]. However, none of these simple bond clcavage pro cesses is apparent in any of the tandem mass spectra obtained under either unimolecular or CID conditions. The combined data thus support the hydride transfer mechanism.

\section{Conclusion}

Two reaction pathways for gas-phase aldose-ketose isomerization were examined using $\Gamma \mathrm{AB}-\mathrm{MS} / \mathrm{MS}$ and semiempirical molecular orbital methods. The theoretical calculations support the hydride transfer mechanism (Pathway B) over that of the 1,3-H shift. The data suggest that the hydride transfer mechanism is favored over dissociation until the stable six-membered transition state can be formed followed by the facile retro-

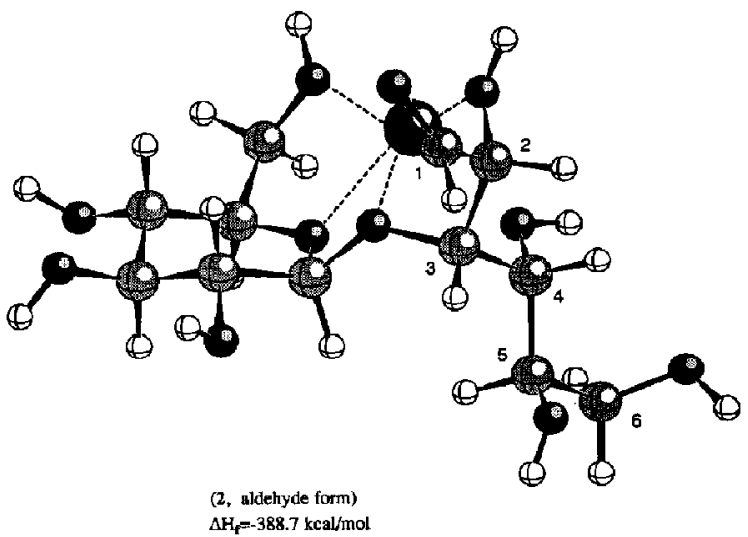

Figure 3. The calculated geometry for the lithium complex of the aldo form (2). 
aldol rearrangement. Because we do not see this same reaction during CID of the protonated precursor, it is very likely that the hydride transfer is being catalyzed by the presence of lithium.

\section{Supplementary Material}

Supplementary material for this article is available in photocopy form from the office of the Editor-in-Chief (see front of journal for address). Requests must include complete title of article, names of authors, issue date, and page numbers. The supplementary material consists of the structures listed below.

Structure 3. Enol intermediate, $\Delta \mathrm{H}_{\mathrm{f}}=-392.0$ $\mathrm{kcal} / \mathrm{mol}$

Structure 4. Keto form, $\Delta H_{f}=-394.3 \mathrm{kcal} / \mathrm{mol}$

Structure 5. TS for enolization process, $\Delta \mathrm{H}_{i}=-311.3$ $\mathrm{kcal} / \mathrm{mol}$

Structure 6. TS for the proton transfer, $\Delta \mathrm{H}_{f}=-332.2$ $\mathrm{kcal} / \mathrm{mol}$

Structure 7. Zwillerionic intermediate, $\Delta \mathrm{II}_{f}--348.6$ $\mathrm{kcal} / \mathrm{mol}$

Structure 8. TS for Hydride transfer, $\Delta \mathbf{H}_{f}=-336.2$ $\mathrm{kcal} / \mathrm{mol}$

Structure 9. $\Delta \mathrm{H}_{\mathrm{f}}=-303.0 \mathrm{kcal} / \mathrm{mol}$

\section{Acknowledgments}

We gratefully acknowledge NIH (ROIGM47356-01) for supporting this research, and also NIH (S10RR03484-01) and NSF (CHE8702669) for shared instrumentation funds for our ZAB2-EQ instrument. We also acknowledge the Graphics Facility at University of California at Berkeley (NIH 850RL05651A) for providing computer time. In addition, we thank Dr. Zhongrui Zhou for her helpful discussions.

\section{References}

1. Zhou, Z.; Ogden, S.; Leary, J. A. J. Org. Chem. 1990, 55, 5444.

2. Hofmeister, G. E.; Zhou, Z.; Leary, J. A. J. Am. Chem. Soc. $1991,113,5964$.
3. Staempfli, A.; Zhou, Z.; Leary, J. A. J. Org. Chem. 1992, 57, 3590.

4. For a general discussion of base-catalyzed reactions of sugars, see: (a) Kieboom, A. P. G.; van Bekkum, H. Recl. Trav. Chim. Pays-Bas 1984, 103, 1; (b) de Bruijn, J. M.; Kieboom, A. P. G.; van Bekkum, H. Sugar Tech. Rev. 1986, 13. 21.

5. Isbell, H. S. Carbohydrates in Solution, no. 117 in a series; American Chemical Society: Washington, DC, 1973, and references therein.

6. Einspahr, H. M., Ed. Protein-Carbohydrate Interactions; American Crystallographic Association, 1989; see also reference 13.

7. Schulenberg, J. W. J. Am. Chem. Soc. 1968, 90, 7008.

8. Saito, S. Chem. Phys. Lett. 1976, 42, 399.

9. (a) Bouma, W. J.; Poppinger, D.; Radom, L. J. Am. Chem. Soc. 1977, 99, 6443; (b) Rodwell, W. R.; Bouma, W. J.; Radom, L Int. J. Quantume Chem. 1980, 18, 107; (c) Smith, B. J.; Nguyen, M. T.; Bouma, W. J.; Radom, L. J. Am. Chem. Soc. 1991, 113, 6452 .

10. Minkin, V. I.; Simkin, B. Y.; Minyaev, R. M. Quantum Chemistry of Organic Compounds; Springer: New York, 1990; p 251.

11. (a) Farber, G. K.; Petsko, G. A.; Ringe, D. Biochemistry 1989, 28, 7289; (b) Farber, G. K.; Petsko, G. A.; Ringe, D. Protein Eng. 1987, 1, 459; (c) Whitlow, M.; Howard, A. J. In ProteinCarbohydrate Interactions; Einspahr, H. M., Ed. American Crystallographic Association, 1989; (d) Whitlow, M.; Howard, A. J.; Fincel, B. C.; Poulos, T. L.; Winborne, E.; Gilliland, G. L Proteins: Struc. Funct. Genet. 1991, 9, 153; (e) Collyer, C. A.; Henrick, K.; Blow, D. M. J. Mol. Biol. 1990, 212, 211; (f) van Tilbeurgh, H.; Jenkins, J.; Chiadmi, M.; Janin, J.; Wodak, S. J.; Mrabet, N. T,; Lambeir, A.-M. Biochemistry 1992, 31, 5467.

12. Zheng, Y.-J.; Merz Jr., K. M.; Farber, G. K. Protein Engineering $1993,6,479$.

13. (a) Ramchander, S.; Feather, M. S. Arch. Biochem. Biophys. 1977, 178, 576; (b) Gleason, W. B.; Barker, R. Can. I. Chem. 1971, 49, 1433; (c) Harris, D. W.; Feather, M. S. J. Am. Chem. Soc. 1975, 97, 178; (d) Ralapati, S.; Feather, M.S. Carbohydrates, Nucleosides, Nucleotides 1978, 5, 297.

14. Dewar, M. J. S.; Thiel, W. J. Am. Chem. Soc. 1977, 99, 4998.

15. Dewar, M. J. S. Org. Mass Spectrom. 1993, 28, 305.

16. Dewar, M. J. S. Int. J. Quantum Chem. 1992, 44, 427.

17. Dewar, M. J. S.; Dieter, K. M. I. Am. Chem. Soc. 1986, 108. 8075 .

18. Dewar, M. J. S.; Zuebisch, E. G.; Healy, E. F.; Stewart, J. J. P. 1. Am. Chem. Soc. 1985, 107, 3902.

19. Wesdemiotis, C.; Fura, A.; McLafferty, F. W. I. Am. Soc. Mass Spectrom. 1991, 2, 459 .

20. Gillece-Castro, B. L.; Burlingame, A. L. Methods in Enzymology 1990, 193, 689.

21. Busch, K. L.; Glish, G. L.; McLuckey, S. A. Mass Spectrometry ' Mass Spectrometry; VCH: New York, 1988.

22. Kerr, J. A. Chem. Rev. 1966, 66, 465. 